\title{
Simultaneous monitoring of separate fetal magnetocardiographic signals in twin pregnancy
}

\author{
S Comani ${ }^{1,2}$, D Mantini $^{3}$, G Alleva $^{1}$, E Gabriele $^{4}$, M Liberati $^{4}$ \\ and G L Romani ${ }^{1,2}$ \\ ${ }^{1}$ Department of Clinical Sciences and Bio-imaging, Chieti University, Italy \\ 2 ITAB-Institute of Advanced Biomedical Technologies, University Foundation \\ 'G D'Annunzio', Chieti University, Italy \\ ${ }^{3}$ Department of Informatics and Automation Engineering, Marche Polytechnic University, \\ Ancona, Italy \\ ${ }^{4}$ Department of Medical Sciences, Chieti University, Italy \\ E-mail: comani@itab.unich.it
}

Received 15 September 2004, accepted for publication 28 January 2005

Published 25 February 2005

Online at stacks.iop.org/PM/26/193

\begin{abstract}
Fetal magnetocardiography (fMCG) allows the non-invasive recording of fetal cardiac electrical activity with increasing efficacy as gestation progresses. Many reports on the successful extraction of reliable fetal magnetocardiographic traces in singleton pregnancies exist in the literature, whereas there is only one report on the reconstruction of averaged fetal cardiac signals obtained in a twin pregnancy with the use of a double sensor array system. In this paper, we aimed at assessing the effectiveness of an ICA-based procedure to reconstruct the time course of fetal cardiac signals recorded with a single-shot multi-channel fMCG device in an uncomplicated twin pregnancy at 27 weeks. The evaluation of heart rate and beats synchronicity permitted the differentiation of fetal components; the quality of reconstructed fetal signals allowed visual inspection on single cycles and the simultaneous monitoring of separate fetal heart rate patterns. The proposed technique might be applied in twin pregnancies not only to characterize fetal arrhythmias, but also in all cases of discordant fetal growth, either in the case of intra-uterine growth retardation affecting one fetus, or in the case of twin-twin transfusion syndrome, a lifethreatening condition where both fetuses are at risk of heart failure.
\end{abstract}

Keywords: fetal magnetocardiography (fMCG), independent component analysis (ICA), cardiac monitoring, prenatal diagnosis 


\section{Introduction}

Recently, fetal magnetocardiography (fMCG) proved to be an efficient tool for the noninvasive detection of fetal electrocardiographic activity and it has been successfully used for the characterization of fetal arrhythmias (van Leeuwen et al 1999, Wakai et al 2000, 2003, Kandori et al 2003, Menendez et al 2001, Hosono et al 2002, Quartero et al 2002, Comani et al 2004a); other clinical applications have been proposed, such as the monitoring of the intra-uterine growth retarded fetus (Grimm et al 2003), cardiac hypertrophy in the fetus of a diabetic mother (Horigome et al 2001), congenital heart defects (Kahler et al 2002) and the monitoring of fetal oxygenation during labor (Lowery et al 2003).

fMCG measures the magnetic field variations related to fetal cardiac function from outside the maternal abdomen. During the second half of pregnancy, fMCG is particularly efficient because, differently from trans-abdominal fECG, it is undistorted by vernix caseosa; moreover, fetal signal intensity, proportional to fetal heart dimensions, undergoes a continuous increase as gestation progresses (Peters et al 2001, Taylor et al 2003).

However, fetal magnetocardiographic signals are mixed with and often hidden by maternal cardiac components and background noise. Consequently, fMCG observations need to be processed in order to reconstruct uncontaminated fetal signals that may be suitable for clinical application. To this aim several techniques have been used, such as the correlation method, which subtracts all maternal QRS complexes from fMCG using a spatio-temporal correlation function (Abraham-Fuchs et al 1990), an eigenvector-based spatial filtering, which isolates the signal from the interference identifying their respective subspaces (Chen et al 2001) or the adaptive maternal beat subtraction procedure, which iteratively eliminates the average maternal signal from fMCG recordings (Comani et al 2004d). However, in the majority of cases averaged signals need to be calculated in order to characterize cardiac waves.

A new procedure, using neural networks, has been proposed by the authors; it is based on an ICA algorithm (FastICA), and it has been successfully applied to reconstruct the time course of fetal magnetocardiographic signals in singleton pregnancies (Comani et al 2004d, 2004e). The retrieved fetal traces were unaffected by maternal interference, and background noise was strongly reduced, so that a high signal-to-noise ratio (SNR) could be attained. The overall good quality and reliability of those signals permitted the measurement of cardiac time intervals and the classification of fetal arrhythmias on single cycles, and not only on averaged beats (Comani et al 2004a, 2004b, 2004d, 2004e).

Although various groups have reported on dependable fetal signal extraction in singleton pregnancy (van Leeuwen et al 1999, Wakai et al 2000, 2003, Kandori et al 2003, Menendez et al 2001, Hosono et al 2002, Quartero et al 2002, Comani et al 2004a, 2004b, 2004d, 2004e, Grimm et al 2003, Horigome et al 2001, Kahler et al 2002, Lowery et al 2003, Peters et al 2001, Stinstra et al 2002), only one case of successful separation of fetal magnetocardiographic signals in twin pregnancy has been reported until present; fMCG was recorded using two distinct MCG sensor arrays positioned over the abdominal projections of the twins' hearts previously obtained with ultrasound examination, and the fetal traces were averaged in time before being analyzed (Costa Monteiro et al 2001).

Conversely, we describe an uncomplicated twin pregnancy at 27 weeks of gestation for which fMCG was performed with a single multi-channel MCG array. In twins, the separation of cardiac signals recorded with a single-shot fMCG can be very difficult not only because their intensities and rhythms are very similar, but also because the dimensions and relative orientations of the fetal hearts may generate unfavorable conditions for an effective fMCG monitoring. 
Table 1. Echocardiographic positions and dimensions of the twins' hearts. All measurements are given in $\mathrm{mm}$.

\begin{tabular}{lll}
\hline & Fetus n.1 & Fetus n.2 \\
\hline Position & $\begin{array}{l}\text { Longitudinal, } \\
\text { vertex, on the } \\
\text { patient's left side }\end{array}$ & $\begin{array}{l}\text { Transverse, with head on the } \\
\text { patient's right side and breech } \\
\text { on the left }\end{array}$ \\
& 101.0 \\
Heart depth $^{\mathrm{a}}$ & 100.0 & 26.7 \\
Heart length $^{\mathrm{b}}$ & 25.2 & 23.2 \\
Heart width $^{\mathrm{c}}$ & 22.3 & \\
\hline a Distance of the fetal atrio-ventricular node from the surface of the maternal abdomen wall. \\
${ }^{\mathrm{b}}$ Distance from apex to base. \\
${ }^{\mathrm{c}}$ Dimension measured transversely at the level of the atrio-ventricular valves.
\end{tabular}

The aim of the present work was to verify whether the ICA-based procedure already tested by the authors for singleton pregnancy could reconstruct the time course of the cardiac activity of both fetuses with the correct rates, morphologies, intensities and polarities, in order to allow a simultaneous monitoring of the twins' cardiac function.

\section{Materials and methods}

\subsection{Case description}

fMCG was performed on a 23-year-old primigravida with a bizygotic pregnancy at 27 weeks of gestation. In bizygotic pregnancies, each fetus has its own placenta and gestational sac, with both the amniotic and chorionic membrane. Both fetuses were normally grown and pregnancy was uncomplicated. Fetal echocardiography, including pulsed and Color Doppler study (AU5 EPI equipment, ESAOTE Biomedica, Italy), was performed to exclude malformations and to assess fetal hearts dimensions, position, orientation and depth with respect to the maternal abdomen wall (table 1). The first fetus was longitudinal, vertex, on the patient's left side, while the second fetus was transverse, with his head on the patient's right side and breech on the left (figure 1).

\subsection{Data acquisition}

The study has been ethically approved, and the patient signed a written informed consent before undergoing fMCG, which was performed with a multi-channel system operating in shielded room. Each magnetic sensor was a low-temperature dc-superconducting quantum interference device- (dc-SQUID) integrated magnetometer kept at liquid helium temperature inside a cylindrical cryostat and detecting magnetic field variations from $0.01 \mathrm{pT}$ to $100 \mathrm{pT}$; general white noise density sensed by a magnetometer was about $5 \mathrm{fT} \mathrm{Hz}^{-1 / 2}$ above $60 \mathrm{~Hz}$ (Della Penna et al 2000). Fifty-five sensors covered a circular area of $415 \mathrm{~cm}^{2}$ and provided 55 simultaneous recordings of the mixed maternal and fetal signals. The fMCG device was positioned above the maternal abdomen, $5 \mathrm{~cm}$ below umbilicus (figure 1), and a singleshot acquisition of 15 min was performed with $1 \mathrm{kHz}$ sampling frequency in the bandwidth $0.016-250 \mathrm{~Hz}$.

\subsection{Data analysis}

In order to reconstruct the time course of the electrocardiographic activity of both fetuses, fMCG recordings were processed with fixed-point independent component analysis (FastICA) 


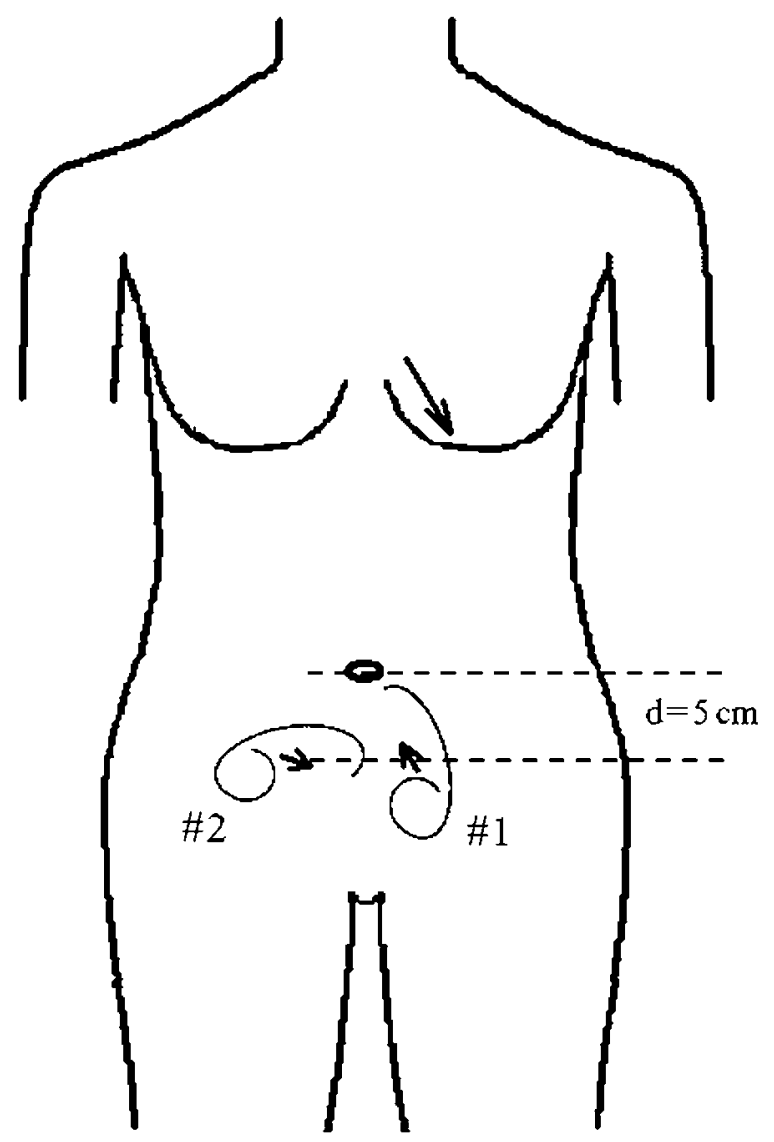

Figure 1. Schematic representation of the positions and orientations of the maternal and fetal hearts derived from ultrasonographic observation. The fMCG device was centered above an ideal line passing through the surface projections of the centers of the fetal hearts; this line was $5 \mathrm{~cm}$ distant from the maternal umbilicus, as indicated. The labels \#1 and \#2 identify the two fetuses inside the maternal abdomen, and the corresponding small arrows specify the orientation of their cardiac axes. The maternal cardiac axis is also represented with an arrow.

(Comon 1994, Bell and Sejnovsky 1995, Hyvärinen 1999, Hyvärinen and Oja 2000); this ICA algorithm was chosen because of its effectiveness and consistency in achieving convergence, as extensively described in the previous papers (Comani et al 2004c, 2004d, 2004e, Mantini et al 2004).

ICA permits a completely blind separation of $m$ signals $s_{i}(t)$ that originate from distinct and independent sources, starting from $n$ observations $x_{j}(t)$ that are linear instantaneous mixtures of $s_{i}(t)$ (Comon 1994, Bell and Sejnovsky 1995). This condition is met in the present case because the sources, i.e., the maternal heart and the twins' hearts, are physically distinct, although producing signals with the same structure. Being ICA based on neural networks, a reliable estimate of the independent components can be achieved when the number of observations $(n)$ is greater than or equal to the number of independent signals $(m)$; this restriction on the number of input traces necessary to retrieve all independent components is satisfied because 55 simultaneous fMCG recordings are available. FastICA applies a fixedpoint iteration rule to estimate all $s_{i}(t)$ (Hyvärinen 1999, Hyvärinen and Oja 2000). 
After all independent components have been retrieved, each of them was ascribed to the appropriate source: maternal components were easily identified because they always showed longer cardiac cycles, whereas fetal components, having a definitely shorter rate, could be differentiated and ascribed to one fetal heart or the other on the basis of cardiac cycles synchronicity. In fact, although twins have similar heart rates and the fetal cardiac rhythm is generally rather variable, ventricular depolarization does not occur simultaneously in twins' hearts: the analysis of QRS synchronicity in different fetal components ensured that they could be correctly differentiated. In general, we may expect to extract a maximum of two components for each fetus. In that case, the components referring to the same fetal heart can be detected because their QRS complexes are always synchronous throughout the trace.

After having identified the components referring to the same fetal heart, the related fetal trace was restored summing the components with their original polarity, which was automatically retrieved using information contained in the mixing matrix generated by the FastICA run; the appropriate signal intensity was attained using information extracted from raw fMCG recordings (Comani et al 2004c). The SNR of the reconstructed fetal signals could be further improved with a smoothing procedure based on a Kaiser filter working with a moving window that maintained the original time resolution (Comani et al 2004d).

Several parameters were calculated: fetal heart rate (FHR), measured in beats per minute (bpm), and its evolution over time during the recording; RR intervals, quantifying the duration of cardiac cycles, measured in milliseconds (ms); SDNN, defined as the standard deviation of all normal sinus RR intervals and representing an estimate of FHR variability, also measured in milliseconds (ms).

\section{Results}

Because of fetal movements during the second half of fMCG acquisition, only the first 8 min of the continuous recording did not present any large or rapid baseline drift and were consequently used for post-processing.

Although only maternal QRS complexes were evident in raw fMCG (see figure 2(a)), the cardiac signals of both fetuses were correctly retrieved, as confirmed by the different heart rates and beats asynchronicity shown in the reconstructed rhythm strips (figures 2(c) and (d)). The SNR of both fetal traces was greater than $6 \mathrm{~dB}$ before smoothing, and greater than $10 \mathrm{~dB}$ after smoothing.

FHR and its beat-to-beat variations could be monitored for both fetuses during the entire 8 min recording: the tachogram of fetus $n .1$ showed a couple of accelerations during the first minutes and a baseline variation followed by a moderate tachycardia after the 4th minute from fMCG beginning, while the tachogram of fetus n. 2 showed a baseline variation followed by a moderate tachycardia occurring around the 6th minute from fMCG beginning (figure 3 ). The analysis of FHR patterns and the visual inspection of fetal signals permitted to check for the presence of ectopic beats, as for singleton pregnancy (Comani et al 2004a, 2004d), but none was found in both fetuses.

Table 2 gives a summary of the basic heart rate parameters (FHR, average RR duration and SDNN) calculated during the 1st, 3rd, 5th and 7th minute. A rhythm increase from 158 to $195 \mathrm{bpm}$ was detected in fetus n.1, with a corresponding SDNN decrease from 22 to $8 \mathrm{~ms}$; similarly, the rhythm of fetus n.2 underwent an increase from 154 to $189 \mathrm{bpm}$, with an SDNN decrease from 16 to $10 \mathrm{~ms}$.

Finally, it is worth noting that the fetal cardiac signals had reversed polarity and that the peak-to-peak amplitude was about $10 \%$ larger in fetus $n .2$ than in fetus $n .1$ (figure 2). Although clear QRS complexes and weaker P and T waves could be distinguished on rhythm 

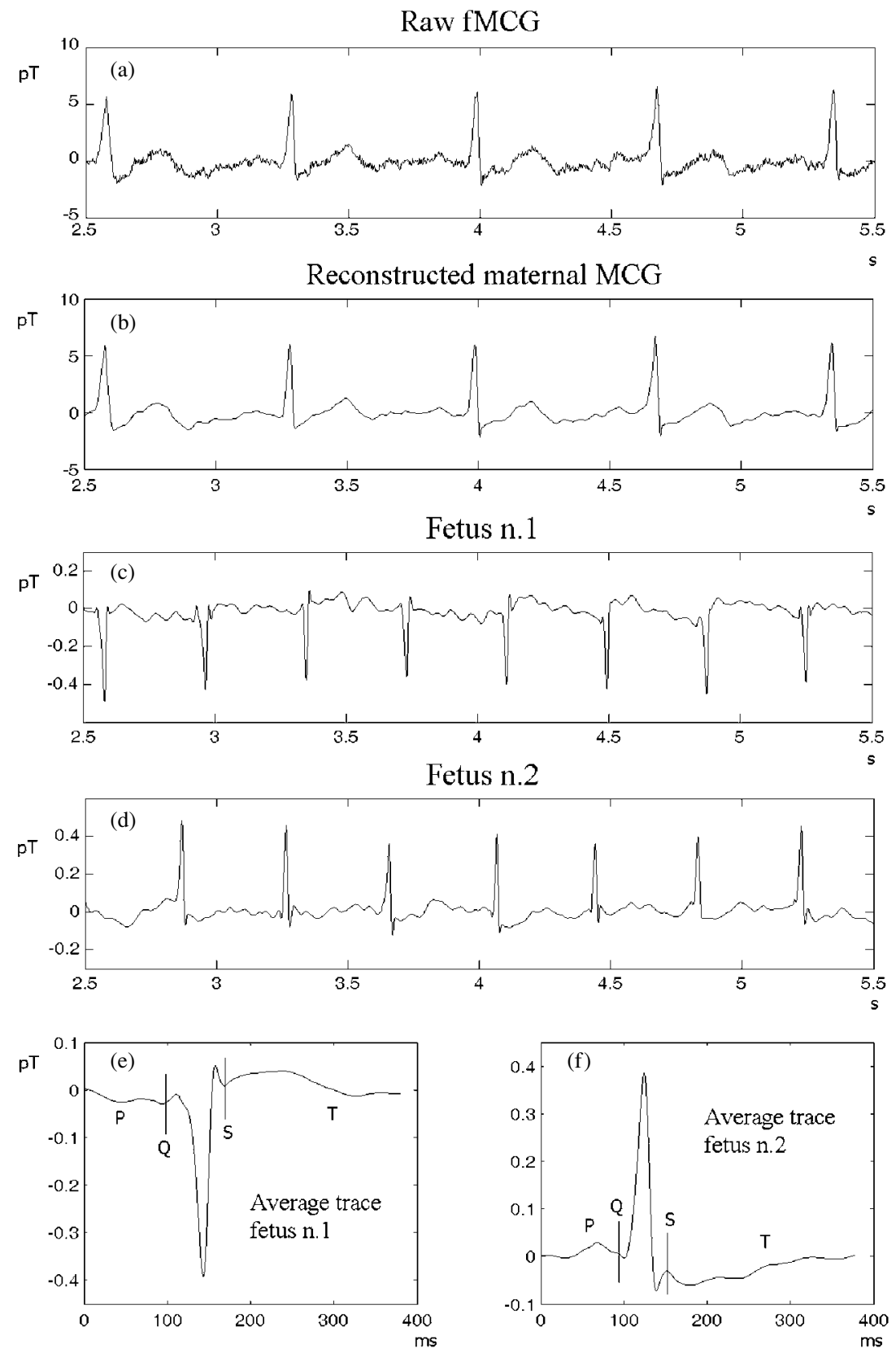

Figure 2. Rhythm strips refer to the 1 st min after the beginning of fMCG recording. (a) Example of real-time fMCG recording where only maternal QRS complexes are evident (magnetic channel n.0); (b) maternal magnetocardiographic trace reconstructed from the signal shown in (a); (c) and (d) simultaneous fetal signals reconstructed with FastICA (fetus n.1 and n.2, respectively); (e) and (f) average sinus beats obtained from signals shown in (c) and (d), respectively. P-QRS-T waves are indicated on averaged traces. Time is expressed in milliseconds and signal intensity in pT.

strips, average beats allowed a better identification of cardiac waves, with $\mathrm{P}$ waves generally better defined than $\mathrm{T}$ waves. 


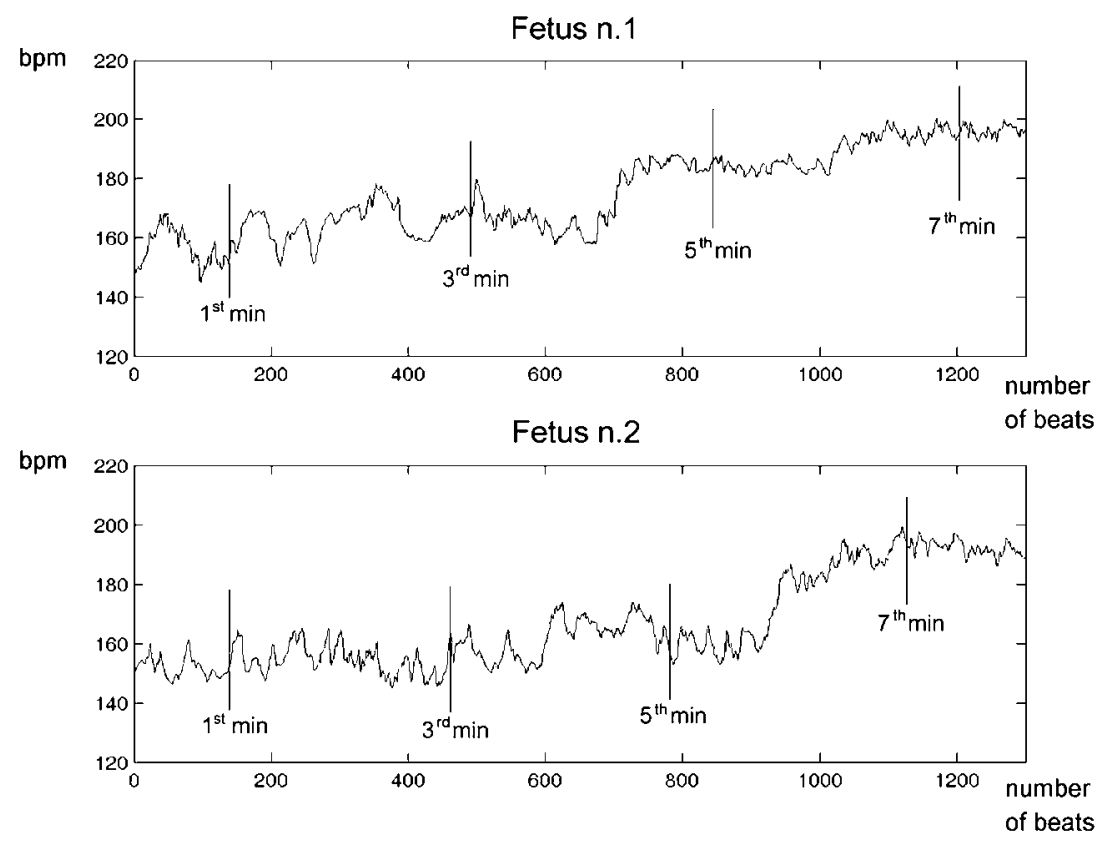

Figure 3. Twins' FHR patterns. FHR is given in beats per min (bpm). Recording duration is expressed in number of beats, 1300 for both fetuses; since FHR of fetuses changed in a different manner during the recording, the length of the tachogram corresponds to $7^{\prime} 30^{\prime \prime}$ for fetus $\mathrm{n} .1$ and to $8^{\prime}$ for fetus n.2. Vertical lines indicate the end of the 1st, 3rd, 5th and 7th min.

Table 2. Evolution of fetal heart rate (FHR) parameters during fMCG acquisition. Averaged values are given at preset intervals, in minutes, from recording beginning.

\begin{tabular}{lrrrr}
\hline Time from fMCG beginning & 1st min & 3rd min & 5th min & 7th min \\
\hline Fetus n.1 & & & & \\
$\quad$ FHR (bpm) & 158 & 169 & 184 & 195 \\
Average RR (ms) & 380 & 355 & 326 & 308 \\
$\quad$ SDNN (ms) & 22 & 21 & 14 & 8 \\
Fetus n.2 & 154 & 151 & 168 & 189 \\
FHR (bpm) & 389 & 397 & 357 & 318 \\
Average RR (ms) & 16 & 15 & 12 & 10 \\
SDNN (ms) & & & & \\
\hline
\end{tabular}

\section{Discussion and conclusion}

The main outcome of this study was the successful separation of magnetocardiographic signals from single-shot fMCG in a twin pregnancy, and the reconstruction of their simultaneous time course and FHR patterns. The use of an ICA-based procedure, which had already provided reliable results in singleton pregnancies (Comani et al 2004a, 2004b, 2004c, 2004d, 2004e), permitted the differentiation of the fetal signals on the basis of heart rate and cardiac cycles synchronicity. The retrieved fetal traces could be ascribed to one fetus or the other using ultrasonographic information gathered prior to fMCG, and their steadiness and clear morphology permitted the simultaneous monitoring of the electrophysiological events occurring in the twins' hearts, impossible on the basis of averaged signals only. 
The difficulties related to the separation of fetal cardiac traces recorded in twin pregnancy with single-shot fMCG, mainly due to their similar intensity and rhythm and to their low peak-to-peak amplitude as compared to signals of age-matched singletons, were successfully overcome with FastICA, basically because it uses neural networks to perform blind signals separation; in fact, neural networks can be extremely effective when the condition on the physical and functional independence of signal sources is satisfied and sufficiently numerous and long simultaneous observations are available, as in the case of multi-channel fMCG.

Several factors support the robustness and effectiveness of the described procedure for the separation of fetal cardiac signals in twin pregnancy: first, the quality of the retrieved fetal traces, which permitted not only to check for the presence of ectopic beats on single cardiac cycles, but also to quantify signal intensity, the latter being lower in fetus n. 1 than in fetus n.2, in agreement with the relative dimensions of the fetal hearts; second, the possibility of monitoring the simultaneous beat-to-beat fluctuations of the twins' heart rate, which highlighted a baseline variation followed by moderate tachycardia in both fetuses, although with a delay of a couple of minutes, as shown in the tachograms.

It is interesting to comment upon the reversed polarities of the fetal signals, which, together with the information obtained with ultrasonography on the orientation of the fetal hearts, allowed associating the reconstructed traces with one fetus or the other. In fact, if we assume that the orientation of the maternal heart produced a positive cardiac signal, the negative and positive polarities of the fetal traces imply that the orientation of the twins' hearts must be, respectively, anti-parallel and parallel to the maternal cardiac axis; therefore, it was possible to associate the fetal signal with negative polarity to fetus n.1, and the other trace to fetus n.2 (figure 1).

It might be argued that the results obtained for one twin pregnancy are not sufficient to draw general conclusions on the effectiveness and reliability of the proposed procedure for the reconstruction of twins magnetocardiographic signals; nonetheless, we believe that its validation on a larger population will confirm these preliminary, very encouraging results, given the quality of the current outcomes, the technical characteristics of FastICA and its performances in singletons (Comani et al 2004a, 2004d).

Trans-abdominal fECG has generally low detection rates during the second half of pregnancy; therefore, the combination of fMCG, which in contrast has good performances, with the described procedure, which led to an effective separation of fetal traces, might be of clinical interest and complement the existing monitoring techniques in several obstetric pathologies.

The separation of fetal magnetocardiographic traces and the monitoring of fetal cardiac rhythms will allow extending to twin pregnancy all the possible clinical indications for fMCG, first of all the characterization of fetal arrhythmias (van Leeuwen et al 1999, Wakai et al 2000, 2003, Kandori et al 2003, Menendez et al 2001, Hosono et al 2002, Quartero et al 2002, Comani et al 2004a), but also situations still under evaluation, such as fetal intra-uterine growth retardation (Grimm et al 2003), cardiac hypertrophy in the fetus of diabetic mother (Horigome et al 2001), congenital heart defects (Kahler et al 2002) and monitoring of fetal oxygenation during labor (Lowery et al 2003).

One particular clinical application of this technique might be the so-called 'twin-twin transfusion syndrome'(TTTS) (Quintero et al 1999). In this syndrome, both fetuses are at risk for heart failure, and timing of delivery is crucial, because it has to be weighted between the risk of prematurity and the risk of intra-uterine death due to the disease. In this pathological condition, the simultaneous monitoring of cardiac traces of both fetuses may highlight signs of cardiac overload and dysfunction, and this might help in optimizing time of delivery. 


\section{References}

Abraham-Fuchs K, Härer W, Schneider S and Stefan H 1990 Pattern recognition in biomagnetic signals by spatiotemporal correlation and application to the localisation of propagating neuronal activity Med. Biol. Eng. Comput. 28 398-406

Bell A J and Sejnovsky T J 1995 An information-maximization approach to blind separation and blind deconvolution Neural Comput. 7 1129-59

Chen M, Wakai R T and Van Veen B 2001 Eigenvector based spatial filtering of fetal biomagnetic signals J. Perinat. Med. 29 486-96

Comani S et al 2004a Characterization of fetal arrhythmias by means of fetal magnetocardiography in three cases of difficult ultrasonographic imaging Pacing Clin. Electrophysiol. 27 1647-55

Comani $\mathrm{S}$ et al 2004b Fetal intra-cardiac intervals for different gestational epochs as evaluated from fetal magnetocardiograms Biomed. Tech. 48 150-2

Comani S, Mantini D, Alleva G, Di Luzio S and Romani G L 2004c Fetal magnetocardiographic mapping using independent component analysis Physiol. Meas. 25 1459-72

Comani S, Mantini D, Lagatta A, Esposito F, Di Luzio S and Romani G L 2004d Time course reconstruction of fetal cardiac signals from fMCG: independent component analysis vs. adaptive maternal beat subtraction Physiol. Meas. 25 1305-21

Comani S, Mantini D, Pennesi P, Lagatta A and Cancellieri G 2004e Independent component analysis: fetal signal reconstruction from magnetocardiographic recordings Comput. Methods Programs Biomed. 75 163-77

Comon P 1994 Independent component analysis_a new concept? Signal Process. 36 287-314

Costa Monteiro E et al 2001 Fetal cardiac activity analysis during twin pregnancy using a multi-channel SQUID system Physica C 354 87-90

Della Penna S et al 2000 Biomagnetic systems for clinical use Phil. Mag. 80 937-48

Grimm B et al 2003 Influence of intrauterine growth restriction on cardiac time intervals evaluated by fetal magnetocardiography Early Hum. Dev. 74 1-11

Horigome $\mathrm{H}$ et al 2001 Detection of cardiac hypertrophy in the fetus by approximation of the current dipole using magnetocardiography Pediatr. Res. 50 242-5

Hosono T et al 2002 The coincidence of fetal magnetocardiography and direct electrocardiography in a case of fetal atrial flutter due to intracardiac tumor Fetal Diagn. Ther. 17 331-3

Hyvärinen A 1999 Fast and robust fixed-point algorithms for independent component analysis IEEE Trans. Neural Netw. 10 626-34

Hyvärinen A and Oja E 2000 Independent component analysis: algorithms and applications Neural Netw. 13 411-30

Kahler C et al 2002 Fetal magnetocardiography in the investigation of congenital heart defects Early Hum. Dev. 69 65-75

Kandori A et al 2003 Classifying cases of fetal Wolff-Parkinson-White syndrome by estimating the accessory pathway from fetal magnetocardiograms Med. Biol. Eng. Comput. 41 33-9

Lowery C L et al 2003 Noninvasive antepartum recording of fetal S-T segment with a newly developed 151-channel magnetic sensor system Am. J. Obstet. Gynaecol. 188 1491-7

Mantini D, Comani S, Pennesi P and Cancellieri G 2004 Tailoring of the independent component analysis to multichannel fMCG recordings for an optimal reconstruction of the fetal cardiac signal Biomed. Tech. 48 186-8

Menendez T et al 2001 Usefulness of magnetocardiography for the investigation of fetal arrhythmias Am. J. Cardiol. $88334-6$

Peters M et al 2001 Monitoring the fetal heart non-invasively: a review of methods J. Perinat. Med. 29 408-16

Quartero H W, Stinstra J G, Golbach E G, Meijboom E J and Peters M J 2002 Clinical implications of fetal magnetocardiography Ultrasound Obstet. Gynaecol. 20 142-53

Quintero R A, Morales W J, Allen M H, Bornick P W, Johnson P K and Kruger M 1999 Staging of twin-twin transfusion syndrome J. Perinatol. $19550-5$

Stinstra J et al 2002 Multicentre study of fetal cardiac time intervals using magnetocardiography Br. J. Obstet. Gynaecol. 109 1235-43

Taylor M J et al 2003 Non-invasive fetal electrocardiography in singleton and multiple pregnancies Br. J. Obstet. Gynaecol. 110 668-78

van Leeuwen $\mathrm{P}$ et al 1999 Magnetocardiography in the diagnosis of fetal arrhythmia Br. J. Obstet. Gynaecol. 106 $1200-8$

Wakai R T et al 2000 Assessment of fetal rhythm in complete congenital heart block by magnetocardiography PACE 23 1047-50

Wakai R T, Strasburger J F, Li Z, Deal B J and Gotteiner N L 2003 Magnetocardiographic rhythm patterns at initiation and termination of fetal supraventricular tachycardia Circulation 107 307-12 